\title{
Plexciton Quenching by Resonant Electron Transfer from Quantum Emitter to Metallic Nanoantenna
}

\author{
D. C. Marinica, ${ }^{\dagger}$ H. Lourenço-Martins, ${ }^{\dagger}$ J. Aizpurua, $^{\ddagger}$ and A. G. Borisov ${ }^{* \dagger, \S}$ \\ ${ }^{\dagger}$ Institut des Sciences Moléculaires d'Orsay, UMR 8214 CNRS-Université Paris-Sud, Bâtiment 351, 91405 Orsay Cedex, France \\ ${ }^{\ddagger}$ Material Physics Center CSIC-UPV/EHU and Donostia International Physics Center DIPC, Paseo Manuel de Lardizabal 5 20018, \\ Donostia-San Sebastián, Spain \\ ${ }^{\S}$ Donostia International Physics Center DIPC, Paseo Manuel de Lardizabal 4 20018, Donostia-San Sebastián, Spain
}

Supporting Information

ABSTRACT: Coupling molecular excitons and localized surface plasmons in hybrid nanostructures leads to appealing, tunable optical properties. In this respect, the knowledge about the excitation dynamics of a quantum emitter close to a plasmonic nanoantenna is of importance from fundamental and practical points of view. We address here the effect of the excited electron tunneling from the emitter into a metallic nanoparticle(s) in the optical response. When close to a plasmonic nanoparticle, the excited state localized on a quantum emitter becomes short-lived because of the electronic coupling with metal conduction band states. We show that as a consequence, the characteristic features associated with the quantum emitter disappear from the optical absorption spectrum. Thus, for the hybrid nanostructure studied here and comprising quantum emitter in the narrow gap of a plasmonic dimer nanoantenna, the quantum tunneling might quench the plexcitonic states. Under certain conditions the optical response of the system approaches that of the individual plasmonic dimer. Excitation decay via resonant electron transfer can play an important role in many situations of interest such as in surface-enhanced spectroscopies, photovoltaics, catalysis, or quantum information, among others.

KEYWORDS: Plexcitons, plasmon, exciton, quantum plasmonics, individual hybrid nanostructures

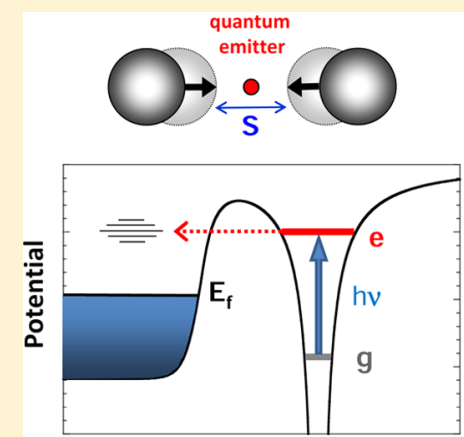

Electron coordinate

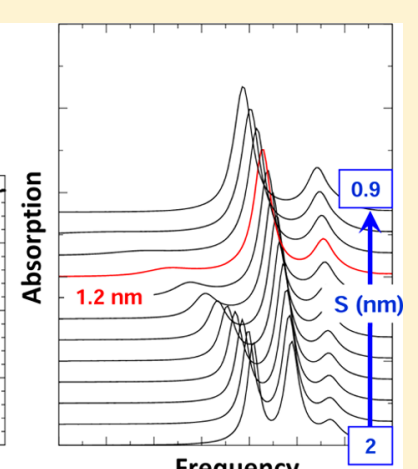

Frequency
B ringing together metallic nanostructures and quantum emitters, such as quantum dots and molecular complexes, offers unprecedented opportunities in controlling light on the nanoscale. Indeed, the tunability of the plasmonic response and of the near field enhancement in metal nanoparticle assemblies $^{1-5}$ allows to engineer the coupling between the excitonic resonance of a quantum emitter (QE) and the collective motion of the metallic electrons, i.e. the plasmons. During the past decade, the study of the interaction between light and these hybrid structures turned into an active field of research ${ }^{6-25}$ owing to its fundamental interest and similarity with cavity quantum electrodynamics, ${ }^{13,19}$ and due to the vast range of possible applications such as sensing, ${ }^{26}$ single photon emitters for information technology, ${ }^{27,28}$ and active devices. $^{29-32}$ With increasing plasmon-exciton coupling strength, when the excitation energy of the emitter is tuned across the plasmon resonance, the Fano profiles in the scattering cross section evolve into the spectral features of avoided crossings ${ }^{13,14,19,20}$ with well-resolved mixed states, so-called plexcitons. ${ }^{21-23}$ As demonstrated in recent experiments, ${ }^{23}$ the near-field enhancement in the junction between nanoparticles $^{3-5,33}$ might lead in this system to the strong plasmon-exciton coupling with large Rabi splitting of the 
efficiency as we discuss below. The subject of quantum effects becomes particularly timely because of the advances in nanofabrication and manipulation techniques. ${ }^{23,37-40}$ In a controllable way, the hybrid nanostructures can be produced with very short separation distances where quantum tunneling and nonlocal screening start to be important in the optical response. Along with offering new opportunities, this technological progress rises new challenges for the theoretical descriptions since conventional classical electrodynamics approaches might be unappropriate. Thus, recent calculations ${ }^{41-44}$ and experiments ${ }^{45,46}$ show that quantum electron tunneling through the narrow junction strongly modifies the near fields and optical response of the plasmonic dimer. When a $\mathrm{QE}$ is located in the tunneling junction, the situation becomes even more complex, and the effect of electron tunneling involving the emitter localized states can strongly affect the response of the whole hybrid system.

In this work, we study the effect of quantum tunneling in the plasmon-exciton hybridization ${ }^{6}$ and thus in the optical response for the canonical case of a $\mathrm{QE}$ placed in the junction of plasmonic dimer. When the $\mathrm{QE}$ is close to the surface of the metallic nanoparticle, the lifetime of the exciton decreases because of an additional decay channel via energy-conserving resonant electron transfer (RET) of the excited electron into the metal. This effect is different from the classical quenching of an emitter's fluorescence close to a metallic surface where the exciton decays into electron-hole pairs via intermediate excitation of a surface plasmon. We show that as a result of RET, for certain conditions and separation distances (typically below $0.6 \mathrm{~nm}$ ) the signature of the $\mathrm{QE}$ in the absorption spectrum can disappear. In particular, in the case of the strong coupling studied here the plexcitonic states are quenched, ${ }^{14}$ and the optical response of the system approaches that of isolated plasmonic dimer. The excited state decay via RET thus sets the lower bound for the distances from the surface where a QE can be detected. In a more general context, our study illustrates the importance of the coupling to the substrate and the resulting reduction of the lifetime of excited electronic states as a limiting factor for photoinduced processes at surfaces. Therefore, our results impact a variety of fields in nanooptics dealing with localized states in the proximity of plasmonic nanoparticles, such as in sensing, catalysis, quantum information, or in active nanooptics involving photochemical processes.

Quantum and Classical Results for a Model System. Our model system is illustrated in Figure 1. Two metallic

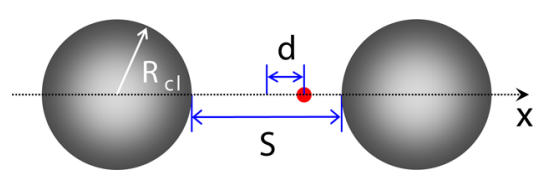

Figure 1. Sketch of geometry of the nanoparticle dimer. Two spherical nanoparticles of radius $R_{\mathrm{cl}}$ are separated by a junction of width $S$. The quantum emitter is placed at the dimer axis ( $x$-axis) at a distance $d$ measured from the center of the junction.

nanospheres of radii $R_{\mathrm{cl}}$ are separated by a junction of width $S$. The QE is located on the interparticle $x$-axis at a distance $d$ measured with respect to the center of the junction. Because we are interested in the effect of the RET between QE and metal nanoparticle(s), and in particular on the modification of the plexcitonic modes in the strong coupling regime, the widths of the junction $S$ considered here are typically below $2 \mathrm{~nm}$.
However, we keep $S$ large enough so that the direct tunneling between metallic nanoparticles is negligible. ${ }^{41-44}$ For such a geometry, besides the RET addressed in this paper, the nonlocal screening can be important. ${ }^{47-50}$ The following strategy is then applied.

(1) Using the full quantum time dependent density functional theory (TDDFT) calculations, ${ }^{51,52}$ we study the plexcitonic coupling in a small hybrid nanostructure in the situation where the RET between $\mathrm{QE}$ and nanoparticles is not allowed. The TDDFT results serve as a benchmark to access the importance of the effects due to nonlocal screening. We find that these are small and show that the classical local electromagnetic approach provides a correct description of the optical response. Computational effort associated with TDDFT imposes to consider a model plasmonic dimer of small $\left(R_{\mathrm{cl}}=2\right.$ $\mathrm{nm}$ ) sodium nanoparticles and to limit the range of the variation of parameters of the system.

(2) Once validated, the classical approach is then applied to study the plasmon-exciton coupling in the system of practical interest; a QE placed in the junction of an Ag plasmonic dimer with $R_{\mathrm{cl}}=8 \mathrm{~nm} \cdot{ }^{13,14}$ Extended calculations of the absorption cross-section are implemented to elucidate the effect of the RET between QE and metal nanoparticles. To this end, the RET is accounted for semiclassically via the self-energy of the excited state, and the evolution of the plexcitonic modes of the system is followed as function of the $S$ and $d$ parameters controlling the separation between the $\mathrm{QE}$ and metal surfaces across the junction.

As explained above, we start with the comparison of the quantum and classical treatments for the small hybrid nanostructure in the absence of RET. Within the TDDFT approach, the two identical metal nanoparticles are described with the spherical jellium model (JM) where the ionic cores of the nanoparticle atoms are represented with uniform background charge density $n_{+}=\left(4 \pi r_{s}^{3} / 3\right)^{-1}$. We use the WignerSeitz radius $r_{\mathrm{s}}$ equal to $4 \mathrm{a}_{0}$ (Bohr radius $\mathrm{a}_{0}=0.053 \mathrm{~nm}$ ) corresponding to $\mathrm{Na}$ metal which is a prototype system for the JM description. The radii of the metal spheres are large enough $R_{\mathrm{cl}}=2.17 \mathrm{~nm}$ to allow for well developed plasmonic modes. While JM lacks the chemical accuracy, it captures the main quantum mechanical effects in the dynamics of conduction electrons in large metal clusters ${ }^{53-55}$ and provides a correct description of collective plasmonic modes for the dimers with narrow junctions in good agreement with recent experiments. $^{41-43,45,46}$

Details on the TDDFT calculations as employed here can be found elsewhere. ${ }^{42}$ Below we only describe the main aspects important for the present study. The time evolution of the electronic density $n(r, t)$ in response to the time-dependent excitation is calculated ab initio within the Kohn-Sham (KS) scheme of the TDDFT. ${ }^{51,52}$ We use the adiabatic local density approximation with the exchange-correlation functional of Gunnarson and Lundqvist. ${ }^{56}$ Retardation effects are neglected due to the small size of the system. The field $\varepsilon(t)$ created by the external perturbation and by the time-dependent charge density of the nanoparticles $n(r, t)$ drives the excitation dynamics of the quantum emitter placed at the middle of the junction. Because of the symmetry we only consider the electric field component along the dimer axis. To separate the role of the nonlocal screening, the hybridization between the electronic states localized on the QE and on the plasmonic nanoparticles is not included at this stage of the study. Thus, the RET does not occur, and we will address its role within a semiclassical 
approach in the next section. Following a widely used methodology, ${ }^{57-59}$ the $\mathrm{QE}$ is then treated as a two level system. As we discuss in detail in the Supporting Information, in the linear response regime the density matrix approach is equivalent to the wave function based treatment with ground $c_{\mathrm{g}}$ and excited state $c_{\mathrm{e}}$ amplitudes evolving in time according to

$$
i \hbar \frac{\mathrm{d}}{\mathrm{d} t}\left(\begin{array}{c}
c_{\mathrm{g}}(t) \\
c_{\mathrm{e}}(t)
\end{array}\right)=\left(\begin{array}{cc}
-\hbar \frac{\Omega}{2} & f(t) \\
f(t) & \hbar \frac{\Omega-i \gamma}{2}
\end{array}\right)\left(\begin{array}{c}
c_{\mathrm{g}}(t) \\
c_{\mathrm{e}}(t)
\end{array}\right)
$$

with initial conditions $c_{\mathrm{g}}(t=0)=1$ and $c_{\mathrm{e}}(t=0)=0$. In eq $1, \Omega$ is the transition frequency from the ground $(g)$ to excited $(e)$ state, $\gamma$ accounts for the population decay and eventual dephasing, and $\left|c_{\mathrm{g}}(t)^{2}\right| \sim 1$ and $\left|c_{\mathrm{e}}(t)\right|^{2}$ is the population of the ground and excited state, respectively. We use $\gamma=0.1 \mathrm{eV}$ so that the TDDFT calculation can be converged within a reasonable time range below 100 fs. The transition dipole matrix elements are given by $f(t)=\overline{\mathrm{e}} \mu \varepsilon(t)$, with $\overline{\mathrm{e}}$ being the electron charge. For the discussion to be quantitative, we use $\mu$ $=0.2 \mathrm{~nm}$ typical for alkali atoms. ${ }^{60,61}$ The dipole moment created on the quantum emitter $p=\overline{\mathrm{e}} 2 \mu \operatorname{Re}\left[c_{g}(t) * c_{e}(t)\right]$ generates the potential acting back on the plasmonic dimer which sets the self-consistent scheme.

In Figure 2, we show the waterfall plot and interpolated image of the absorption cross section $\sigma(\omega)$ of the hybrid nanostructure calculated with TDDFT (left panels). Results are shown as function of the frequency $\omega$ of the incoming $x$ polarized plane wave for different transition frequencies $\Omega$ of the $\mathrm{QE}$ placed at the center of a $1 \mathrm{~nm}$ wide junction. When $\Omega$ is far from the resonance with the bonding dipole plasmon (BDP) and the bonding quadrupole plasmon (BQP) modes of the dimer ${ }^{33}$ at $\omega_{\mathrm{BQP}}=3.2 \mathrm{eV}$ and $\omega_{\mathrm{BDP}}=2.8 \mathrm{eV}$, the optical response of the hybrid nanostructure is characterized by wellseparated resonant features. Resonance at $\Omega$ is due to the excitation of the QE. The higher frequency part of $\sigma(\omega)$ is close to that of the isolated dimer and reflects the excitation of the $\mathrm{BDP}$ and higher plasmonic modes. As the transition frequency $\Omega$ is tuned into the resonance with BDP, the exciton and BDP hybridize into the plexitonic states with large Rabi splitting. ${ }^{12-15,23}$ The spectral structure of avoided crossing is particularly well seen in the lower left panel of Figure 2, and it is characteristic for the strong coupling regime attained here because of the field enhancement in the junction.

Note that in the strong coupling regime the decay rate $\gamma$ of the QE has little effect on the avoided crossing structure, as far as $\gamma$ is small. Indeed, when the QE exciton is resonant with the BDP of the dimer, $\Omega=\omega_{\mathrm{BDP}}$, the complex frequencies of the plexcitonic states can be estimated from the two state model ${ }^{13}$ which gives $\Omega_{ \pm}=\Omega-i\left(\gamma+\gamma_{\mathrm{BDP}}\right) / 4 \pm\left(V^{2}-\left(\gamma-\gamma_{\mathrm{BDP}}\right) /\right.$ $16)^{1 / 2}$, where $V$ is the BDP-exciton coupling rate. In our system, the width of the BDP $\gamma_{\mathrm{BDP}}=0.22 \mathrm{eV}$ is at least twice larger than the decay rate $\gamma$ used for the $\mathrm{QE}$, and the analysis of the results shows that the coupling strength $V \simeq 0.3 \mathrm{eV}$. Thus, this is primarily the large coupling strength $V$ that sets $\Omega_{+}$and $\Omega_{-}$. We further discuss these issues in the Supporting Information. The two-state model predicts equal widths of the plexcitonic modes at $\Omega=\omega_{\mathrm{BDP}}$, which is only approximately verified by the present results because of the coupling to the $\mathrm{BQP}$ and higher order modes. ${ }^{14}$ For $\Omega \geq 3.5 \mathrm{eV}$ above the $B Q P$ of the dimer, the absorption spectrum shows the lowerfrequency branch, but the high-frequency structure associated
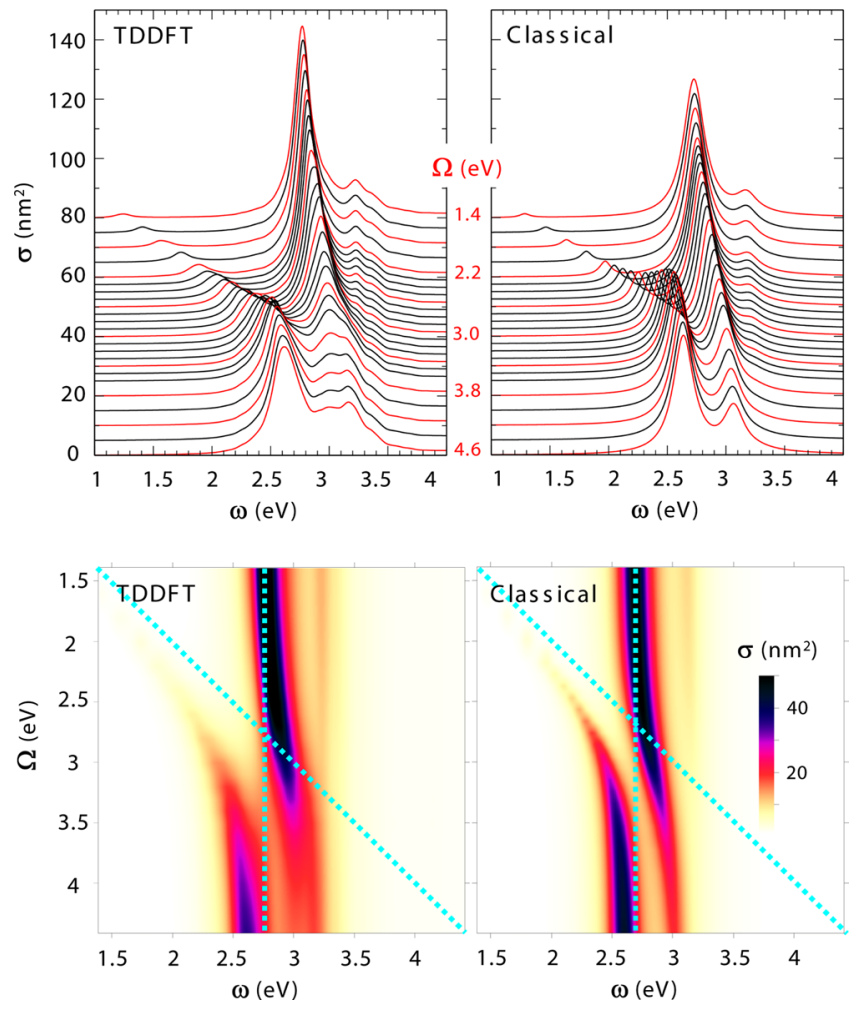

Figure 2. (Upper panels) Waterfall plot of the absorption crosssection obtained in full quantum TDDFT (left) and classical (right) calculations of the hybrid structure with $S=1 \mathrm{~nm}$ and $d=0$ (QE at the center of the junction). Results are shown as function of the frequency $\omega$ of the incoming $x$-polarized plane wave for different frequencies of the quantum emitter $\Omega$. The $\Omega$-values grow in steps of $0.2 \mathrm{eV}(0.1 \mathrm{eV}$ in the avoided crossing region), and the red curves are inserted every $0.4 \mathrm{eV}$. (Lower panels) The same results are shown as interpolated images in the $(\omega, \Omega)$ plane allowing for a clear representation of the avoided crossing structure between the bonding dipole plasmon mode of the nanosphere dimer (vertical dashed blue line) and the $\mathrm{QE}$ exciton (dashed blue line with $\omega=\Omega$ ).

with excitonic transition is absent. This is consistent with the small fields in the junction of an isolated dimer as we calculate for the frequency of external field well above BDP and BQP resonances. Thus, in the hybrid $\mathrm{QE}-$ dimer nanostructure the excitation of the $\mathrm{QE}$ becomes too small to give a visible signature in the spectra.

Along with TDDFT data we also show in Figure 2 (right panel) the absorption cross section calculated with quasi-static local classical approach similar to the one reported in ref 12 . The metallic nanospheres are modeled with Drude permittivity $\varepsilon(\omega)=1-\omega_{\mathrm{p}}^{2} / \omega(\omega+i \eta)$. The plasma frequency $\omega_{\mathrm{p}}=5.16 \mathrm{eV}$ and attenuation $\eta=0.22 \mathrm{eV}$ are set such that for the isolated dimer classical results for $\sigma(\omega)$ match the results from the TDDFT calculations. ${ }^{42}$ The $\mathrm{QE}$ is modeled as a point polarizable object with frequency dependent polarizability: $\alpha(\omega)=2 \Omega \mu^{2} / \hbar\left(\Omega^{2}-\omega^{2}-i \gamma \omega\right)$. It follows from Figure 2 that the TDDFT results are well reproduced with the local classical calculations. The remaining minor differences between the two approaches are primarily due to the quantum finite size effects in the plasmonic dimer. These can not be captured with a classical model and are present already without the interaction with the QE. In particular, the coupling of the plasmon mode to the electron-hole pair excitations ${ }^{44,54,55}$ leads to the structures in the BQP peak. The overall good quantum-classical 

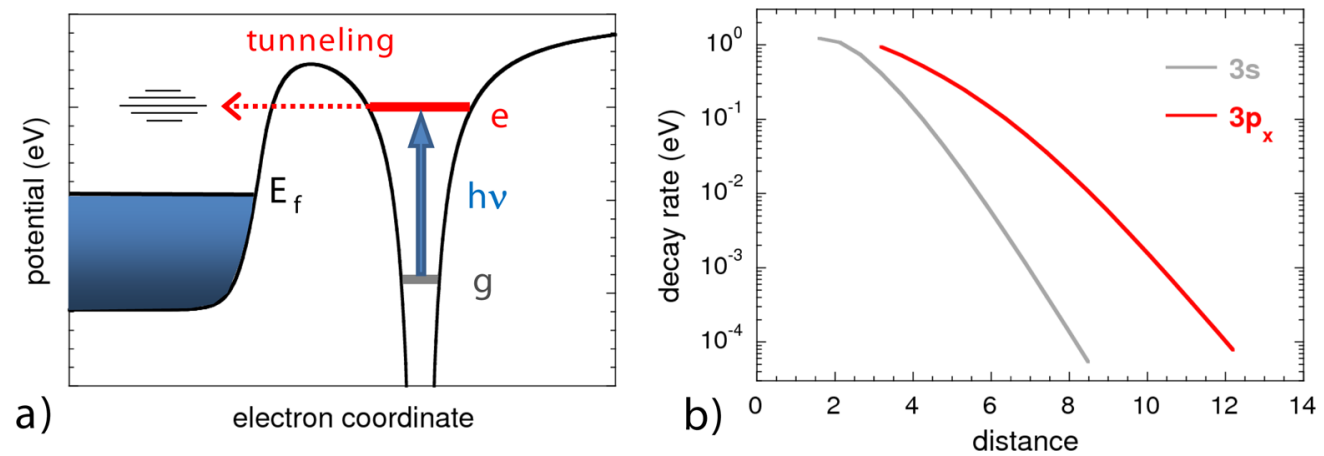

Figure 3. (a) Sketch of the excitation quenching via RET between a QE and a metal nanoparticle. The effective potential "seen" by the active electron is shown along the $x$-axis perpendicular to the metal surface and passing through the QE center. Blue arrow indicates the photon absorption producing an electronic excitation from the ground $(\mathrm{g})$ to the excited (e) electronic state localized in the QE potential well. The red dashed arrow indicates the tunneling of the excited electron through the potential barrier between the QE and the metal. Equivalently, it corresponds to the RET of the excited electron from the discrete state (e) of the QE into continuum of unoccupied electronic states of the metal above Fermi level $E_{f}$ b) The rates of the one electron tunneling between the $\mathrm{Na}$ atom and the free-elctron metal surface. Results are shown as function of the atom-surface distance for the ground state $(3 s)$ and excited state $\left(3 p_{x}\right)$ oriented perpendicular to the nanoparticle surface.

correspondence in the studied model system shows that the nonlocal screening effects are small. It is noteworthy that because of the smallness of the nonlocal effects the classical theory should be also adequate in the perturbative weak coupling regime where the QE induces a narrow Fano profile in the absorption cross-section of the hybrid nanostructure. ${ }^{13,14}$ The above results allow us to use the classical approach for the description of the RET between a $\mathrm{QE}$ and a plasmonic nanoparticle in a more realistic system and for a wide range of geometries, which would be out of reach within TDDFT calculations.

Tunneling of the Excited Electron into Plasmonic Nanoparticle. In the proximity of the metal surface, excited electronic states localized on a QE (quantum dot, molecule, or atom) can decay via electron tunneling into the metal bulk. ${ }^{6}$ This process of RET, particularly well studied in the context of the projectile-surface charge transfer, ${ }^{62-64}$ and in the timeresolved two-photon-photoemission (TR-2PPE) from absorbed species $^{35,65-67}$ is schematically represented in Figure 3a. An electron promoted from the ground state $(g)$ into the excited state $(e)$ via photon absorption, tunnels through the potential barrier between the QE and the metal and escapes into the continuum of propagating electronic states of the metal bulk above the Fermi level. ${ }^{35}$ Because of the discrete statecontinuum coupling, the excited state appears as a resonance in the electronic structure of the interacting system with a width given by the RET rate $\Gamma(L)$, for a given distance $L$ between the QE and the metal suface. Because of the higher binding energy, the ground state is much less coupled to the metal and can still be considered as stationary for the $L$ distance range of interest here.

The above discussion is further supported by the results shown in Figure $3 \mathrm{~b}$ for a $\mathrm{Na}$ atom in front of the free electron metal surface. The RET rates for the (3s) ground state and excited $\left(3 \mathrm{p}_{x}\right)$ state oriented perpendicular to the surface are shown as function of the atom-surface distance $L$. With decreasing $L$ the tunneling barrier between the atom and the metal narrows, so that $\Gamma(L)$ grows as $\Gamma(L) \approx \Gamma_{0} \exp \left(-L / L_{0}\right)$. Observe that in overall the RET rate of the $3 \mathrm{p}_{x}$ state is much larger than that of the $3 \mathrm{~s}$ ground state, where the latter is characterized by a smaller $L_{0}$ parameter because of the higher wave function localization. The decay rates shown in Figure $3 \mathrm{~b}$ were obtained in full quantum calculations ${ }^{63,64}$ and allow for a quantitative description of the charge transfer in ion beam scattering at surfaces as well as of the TR-2PPE data. ${ }^{62,64,67}$ Thus, we made a choice of using typical parameters calculated for alkali atoms to set the realistic distance ranges where the tunneling process can affect the plasmon-exciton coupling (the optical response of the hybrid nanostructure). Below in this paper, we discuss the robustness of our conclusions with respect to the exact nature of the QE.

To reveal the role of the RET we consider the hybrid nanostructure, where the model $\mathrm{QE}$ placed in the junction between $R_{\mathrm{cl}}=8 \mathrm{~nm}$ spherical silver nanoparticles. This geometry and material choice allows for direct comparison with earlier studies in similar systems. ${ }^{13,14}$ In Figure 4, we show the results of classical calculations of the absorption cross section performed with and without account for the excitedstate decay via RET into the metal nanoparticle(s). The Ag nanoparticles are described with a frequency-dependent Johnson and Christy dielectric function. ${ }^{68}$ The polarizability of the model $\mathrm{QE}$ is given by

$$
\alpha(\omega)=\frac{1}{\hbar} \frac{2 \mu^{2} \Omega}{\Omega^{2}-\omega^{2}-i\left(\gamma+\Gamma_{\mathrm{RET}}\right) \omega}
$$

where we use typical values of $\mu=0.3 \mathrm{~nm}$ and $\gamma=50 \mathrm{meV}$, as reported in the literature for J-aggregates. ${ }^{12,13,21,23,31}$ Since the curvature radii of plasmonic nanoparticles are much larger than the distances between the QE and metal surface where the RET is important, the RET rate can be calculated within the flat surface approximation: $\Gamma_{\mathrm{RET}}=\Gamma(S / 2-d)+\Gamma(S / 2+d)$, where $\Gamma(L)$ is the distance dependent decay rate of the $3 \mathrm{p}_{x}$ state as shown in Figure $3 \mathrm{~b}$. With $\Gamma_{\mathrm{RET}}=0$, one retrieves the conventional approach without account for electron tunneling. The $\mathrm{QE}$ excitation frequency $\Omega=3.45 \mathrm{eV}$ is set close to the frequency of the dimer BDP so that the plexcitonic states are well resolved in the absorption cross section. The upper panels of Figure 4 correspond to the situation where the QE is placed at the middle of the junction, and the junction width is progressively reduced from 2 to $0.9 \mathrm{~nm}$. Lower panels correspond to the fixed junction width of $2 \mathrm{~nm}$ and the $\mathrm{QE}$ is progressively moved along the dimer axis from the center of the junction toward one of the nanospheres. In all cases, the junction width $S$ is such that the direct tunneling between plasmonic nanoparticles is negligible. ${ }^{42}$ 

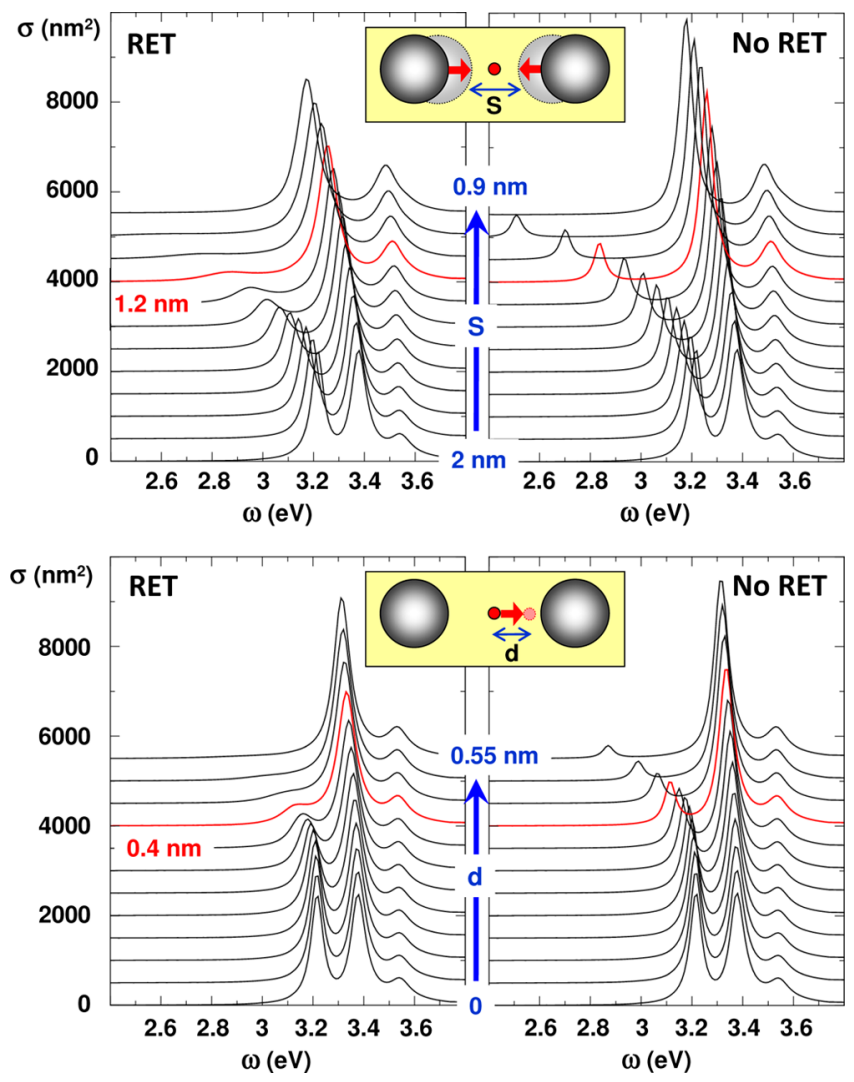

Figure 4. Waterfall plot of the absorption cross-section obtained from classical calculations with (left panels) and without (right panels) inclusion of the RET from the QE into the plasmonic nanoparticles. Results are shown as function of the frequency $\omega$ of the incoming $x$ polarized plane wave, and the QE excitation frequency is set as $\Omega=$ $3.45 \mathrm{eV}$. The plasmonic dimer is formed by $R_{\mathrm{cl}}=8 \mathrm{~nm} \mathrm{Ag}$ nanospheres. (Upper panels) Evolution of the absorption cross section upon change of the junction width $S$ from 20 to $9 \AA$ in steps of $1 \AA$. The QE is at the center of the junction, $d=0$. The arrow indicates the direction of the $S$-decrease. (Lower panels) Evolution of the absorption cross section upon displacement of the QE from the center of the junction toward one of the nanoparticles. The values of $d$ grow from 0 to $5.5 \AA$ in steps of $0.5 \AA$. The junction width $S$ is fixed $S$ $=20 \AA$. The arrow indicates the direction of the $d$-increase.

When the QE is placed in the middle of the junction, the system is in the strong plasmon-exciton coupling regime with large Rabi splitting of plexcitonic states. ${ }^{13,14}$ Similar results have been reported in recent experiments. ${ }^{23}$ Despite the work of Schalter et $\mathrm{al}^{23}$ has been performed for an individual metallic dimer surrounded by a layer of J-aggregates, the authors demonstrate the decisive role of the molecules located in the junction and thus subjected to the strong plasmonic field enhancement. In our calculations shown in the right panels of Figure 4, that is, neglecting the RET into the metal, two plexciton modes are always distinguishable for the variation of the system geometry as considered in the present work. Thus, maintaining the QE at the middle of the junction and reducing the junction width $S$ (upper panel) leads to an overall red shift of the modes and even larger Rabi splitting reflecting the increasing plasmon-exciton coupling. This is because reducing $S$ results in a stronger attractive interaction of the plasmon induced charges across the junction, and in a larger field enhancement at the position of the QE. ${ }^{3-5,33}$ When the junction width $S$ is fixed, and the QE is moved toward one of the nanospheres (lower panels), the results vary only little for 0 $\leq d \leq 0.2 \mathrm{~nm}$. Indeed, for fixed $S$, the interaction between plasmon induced charges across the junction does not change, and because the plasmon induced fields are rather homogeneous along the dimer axis, the plasmon-exciton coupling does not change either. Further increase in $d$ breaks the symmetry of the system and favors the interaction between the point dipole and the closest nanosphere. The lower frequency plexcitonic branch with more exciton character shifts to lower frequencies. Simultaneously, the upper branch evolves into the BDP peak of the dimer.

As follows from Figure 4 (left panels), accounting for the quantum tunneling from the excited state into the metal leads to qualitatively different results. Typically, when the separation between the QE and either of the metal surfaces across the junction reaches the threshold value $L_{\text {th }} \approx 0.6 \mathrm{~nm}$, the lowest frequency plexciton branch with dominant exciton character quickly broadens and looses its intensity. The upper frequency plexciton branch evolves into the BDP resonance, and the entire absorption spectrum approaches that of the isolated $\mathrm{Ag}$ dimer. The plexcitonic states are thus destroyed at QE-metal surface separations corresponding to the threshold RET rate $\Gamma_{\mathrm{RET}}^{\mathrm{th}}$ such that the total exciton line width $\gamma+\Gamma_{\mathrm{RET}}^{\mathrm{th}} \simeq 0.3 \mathrm{eV}$, that is, of the order of magnitude of the Rabi splitting $\Omega_{R}$ between the plexciton frequencies. Indeed, in order to see the complete oscillation with excitation exchange between plasmon and exciton in time domain, ${ }^{25}$ one needs that the latter is preserved on the corresponding time scale given by the $2 \pi$ / $\Omega_{\mathrm{R}}{ }^{13}$ It is noteworthy that for $\gamma$ within some tens of millielectronvolts range, its precise value has little effect on $L_{\text {th }}$. The latter is mainly determined by the RET rate $\Gamma_{\text {RET }}(L)$, which grows exponentially with decreasing $L$, and reaches the values in the electronvolts range when the $\mathrm{QE}$ is close to the nanoparticle surface. As a summary of the results presented in Figure 4, the decay of the excited state via an electron tunneling into the metal bulk profoundly modifies the absorption spectra of the hybrid nanostructure comprising the $\mathrm{QE}$ and the plasmonic dimer. When the potential barrier separating the QE from the metal nanoparticle becomes low enough only the plasmon resonances due to metal nanoparticle(s) are observed.

It is interesting to compare (i) the RET mechanism of the total quenching of the excited state discussed here and active for the QE distances from the metal surface below typically 0.6 $\mathrm{nm}$ with (ii) the quenching of excited state fluorescence found in the classical calculations for QE distances from the metal surface below typically $2 \mathrm{~nm}$. In case (ii), the nonradiative decay dominates the excited state dynamics. Because of the electromagnetic coupling the energy is transferred to plasmon excitations with their subsequent decay into the electron-hole pairs. The QE still appears in the optical absorption spectrum through the Fano resonance or plexciton splitting as in the present study. In case (i) when the RET is fast, the excitation of the $\mathrm{QE}$ quickly decays because of the electronic coupling between the QE localized excited electronic states and the continuum of the electronic states of the nearby metal. The excited electronic state of the QE merges into the continuum of the bulk electronic states, and the quantum emitter related features disappear not only in the fluorescence but in the optical absorption. While plasmonic quenching of fluorescence has been addressed successfully involving classical electromagnetic interactions, ${ }^{39,40,69,70}$ the RET mechanism requires a quantum mechanical calculation of the electronic hybridization between the QE and the metal. 
Several comments are in order regarding the model description used in this work and the generality of the results. Despite present calculations that have been performed with a model QE using RET rates as calculated for a $\mathrm{Na}$ atom, the qualitative results are robust and are caused by universal quantum mechanical phenomena such as electron tunneling. Indeed, any atomic or molecular specie brought close enough to the metal surface couples to the continuum of propagating electronic states of the conduction band. ${ }^{6,35,62,65-67}$ To this regard, the exciton decay via electron tunneling ${ }^{6,35}$ reflects coupling between localized and continuum electronic states, and it is of the same nature as, for example, the excited state broadening effects in surface-enhanced Raman scattering ${ }^{71,72}$ and electron transport through molecular junctions. ${ }^{73}$ The coupling strength depends on the particular system, however, the values of the RET rates used here are typical for the atomic and molecular species interacting with metal surfaces as confirmed in a vast number of experimental and theoretical studies. $^{35,62,65-67}$ This said, the decay rate may be reduced for extended excitonic systems, where an electron spends a long time inside the system before attempting to cross the potential barrier into the metal. Finally, we have neglected the excitedstate population relaxation linked with many body interactions at surfaces such as electron-electron scattering. It has been shown however that many-body relaxation channels are much less efficient than one electron processes. ${ }^{67}$ All the above sets strong confidence on the generality of the results presented here.

Conclusions. In conclusion, we have studied the effect of the resonant electron tunneling on the optical response of the hybrid nanostructure consisting of a QE and a metallic nanoantenna. Our results demonstrate that when the distance between the $\mathrm{QE}$ and the metal surface of plasmonic nanoparticle(s) is below typically $0.6 \mathrm{~nm}$, the QE localized excited electronic states become short lived because of the RET of the excited electron into the metal bulk. As a result, the signature of the $\mathrm{QE}$ in the optical absorption spectrum disappears. For the $\mathrm{QE}$ in the gap of a plasmonic dimer addressed in the present study and characterized by the strong plasmon-exciton coupling, the plexcitonic states are destroyed and the absorption spectrum of the hybrid nanostructure is close to that of the individual plasmonic dimer. For the case of weak coupling, a large resonance width of the QE would lead to the disappearance of the Fano profile in the optical absorption spectrum as can be inferred from the analysis reported in ref 14 . Thus, in addition to the well studied fluorescence quenching occurring for distances between the QE and metal nanoparticle of a few nanometers (due to the transfer of the excitation to plasmons with the subsequent decay to electron-hole pairs), ${ }^{39,40,69,70}$ we have shown that the signature of QE can be erased also in the optical absorption spectra because of the direct escape of the excited electron via quantum tunneling into the metal. The mechanism discussed here is general and involves the RET between the $\mathrm{QE}$ and the nanoparticle(s). The RET does not require a particular dimer geometry and it is effective even for individual plasmonic nanoparticles or for wide junctions. It thus sets the limits for the hybrid nanostructure geometry allowing for the observation of the plexciton states, and more generally for the observation of the spectral features due to the QE. For the layered structures comprising plasmonic nanoparticles and molecular materials or quantum dots, our results imply that the quantum emitters closest to the metal surface might have no effect on the optical response. Since the rates of the RET grow exponentially with decreasing QE-metal surface distance, typically reaching the range of electronvolts for adsorption distances, ${ }^{35,62,67}$ the phenomena described here should be considered in many applications of nanooptics involving electronically excited states.

\section{ASSOCIATED CONTENT}

\section{S Supporting Information}

The details on the wave function and density matrix treatment of the excitation dynamics in QE. The effect of the intrinsic decay rate of the QE on the Rabi splitting of the plexcitonic states calculated with TDDFT. This material is available free of charge via the Internet at http://pubs.acs.org.

\section{AUTHOR INFORMATION}

\section{Corresponding Author}

*E-mail: andrei.borissov@u-psud.fr.

\section{Notes}

The authors declare no competing financial interest.

\section{ACKNOWLEDGMENTS}

Discussions with P. Nordlander are gratefully acknowledged. J.A. acknowledges financial support from the Department of Industry of the Basque Government through the ETORTEK project nanoiker, the Department of Education of the Basque Government through project IT756/13 for consolidated groups, and the Spanish Ministerio de Ciencia e Innovación through Project No. FIS2010-19609-C02-01. This work was performed using HPC resources from GENCI-[TGCC/IDRIS] (Grant x2013057060).

\section{REFERENCES}

(1) Kelly, L.; Coronado, E.; Zhao, L. L.; Schatz, G. C. J. Phys. Chem. B 2003, 107 (3), 668-677.

(2) Alvarez-Puebla, R.; Liz-Marzán, L. M.; García de Abajo, F. J. J. Phys. Chem. Lett. 2010, 1 (16), 2428-2434.

(3) Schuller, J. A.; Barnard, E. S.; Cai, W.; Jun, Y. C.; White, J. S.; Brongersma, M. L. Nat. Mater. 2010, 9, 193-204.

(4) Halas, N. J.; Lal, S.; Chang, W.-S.; Link, S.; Nordlander, P. Chem. Rev. 2011, 111 (6), 3913-3961.

(5) Pasquale, A. J.; Reinhard, B. M.; Negro, L. D. ACS Nano 2011, 5 (8), 6578-6585.

(6) Wiederrecht, G. P.; Wurtz, G. A.; Hranisavljevic, J. Nano Lett. 2004, 4 (11), 2121-2125.

(7) Neuhauser, D.; Lopata, K. J. Chem. Phys. 2007, 127, 154715.

(8) Wurtz, G. A.; Evans, P. R.; Hendren, W.; Atkinson, R.; Dickson, W.; Pollard, R. J.; Zayats, A. V.; Harrison, W.; Bower, Ch. Nano Lett. 2007, 7 (5), 1297-1303.

(9) Trügler, A.; Hohenester, U. Phys. Rev. B 2008, 77, 115403.

(10) Juluri, B. K.; Lu, M.; Zheng, Y. B.; Huang, T. J.; Jensen, L. J. Phys. Chem. C 2009, 113, 18499-18503.

(11) Hakala, T. K.; Toppari, J. J.; Kuzyk, A.; Pettersson, M.; Tikkanen, H.; Kunttu, H.; Törmä, P. Phys. Rev. Lett. 2009, 103 (5), 053602 .

(12) Govorov, A. O.; Bryant, G. W.; Zhang, W.; Skeini, T.; Lee, J.; Kotov, N. A.; Slocik, J. M.; Naik, R. R. Nano Lett. 2006, 6 (5), 984994.

(13) Savasta, S.; Saija, R.; Ridolfo, A.; Di Stefano, O.; Denti, P.; Borghese, F. ACS Nano 2010, 4 (11), 6369-6376.

(14) Manjavacas, A.; García de Abajo, J.; Nordlander, P. Nano Lett. 2011, 11 (6), 2318-2323.

(15) Cohen-Hoshen, E.; Bryant, G. W.; Pinkas, I.; Sperling, J.; BarJoseph, I. Nano Lett. 2012, 12 (8), 4260-4264.

(16) Gómez, D. E.; Roberts, A.; Davis, T. J.; Vernon, K. C. Phys. Rev. B 2012, 86 (3), 035411. 
(17) Pérez González, O.; Aizpurua, J.; Zabala, N. Optics Express 2013, 21 (13), 15847.

(18) Alpeggiani, F.; D’Agostino, S.; Andreani, L. C. Phys. Rev. B 2012, $86(3), 035421$.

(19) Ridolfo, A.; Di Stefano, O.; Fina, N.; Saija, R.; Savasta, S. Phys. Rev. Lett. 2010, 105 (26), 263601.

(20) Ni, W.; Ambjörnsson, T.; Apell, S. P.; Chen, H.; Wang, J. Nano Lett. 2010, 10 (1), 77-84.

(21) Fofang, N. T.; Park, T. H.; Neumann, O.; Mirin, N. A.; Nordlander, P.; Halas, N. J. Nano Lett. 2008, 8 (10), 3481-3487.

(22) Fofang, N. T.; Grady, N. K.; Fan, Z.; Govorov, A. O.; Halas, N. J. Nano Lett. 2011, 11 (4), 1556-1560.

(23) Schlather, A. E.; Large, N.; Urban, A. S.; Nordlander, P.; Halas, N. J. Nano Lett. 2013, 13 (7), 3281-3286.

(24) Lawrie, B. J.; Kim, K.-W.; Norton, D. P.; Haglund, R. F. Nano Lett. 2012, 12 (12), 6152-6157.

(25) Vasa, P.; Wang, W.; Pomraenke, R.; Lammers, M.; Maiuri, M.; Manzoni, C.; Cerullo, G.; Lienau, Ch. Nat. Photonics 2013, 7, 128132.

(26) Lee, J.; Hernandez, P.; Lee, J.; Govorov, A. O.; Kotov, N. A. Nat. Mater. 2007, 6 (4), 291-295.

(27) Taminiau, T. H.; Stefani, F. D.; Segerink, F. B.; van Hulst, N. F. Nat. Photonics 2008, 2 (4), 234-237.

(28) Belacel, C.; Habert, B.; Bigourdan, F.; Marquier, F.; Hugonin, J.P.; Michaelis de Vasconcellos, S.; Lafosse, X.; Coolen, L.; Schwob, C.; Javaux, C.; Dubertret, B.; Greffet, J.-J.; Senellart, P.; Maitre, A. Nano Lett. 2013, 13 (4), 1516-1521.

(29) Pacifici, D.; Lezec, H. J.; Atwater, H. A. Nat. Photonics 2007, 1 (7), 402-406.

(30) Pala, R. A.; Shimizu, K. T.; Melosh, N. A.; Brongersma, M. L. Nano Lett. 2008, 8 (5), 1506-1510.

(31) Vasa, P.; Pomraenke, R.; Cirmi, G.; De Re, E.; Wang, W.; Schwieger, S.; Leipold, D.; Runge, E.; Cerullo, G.; Lienau, C. ACS Nano 2010, 4 (12), 7559-7565.

(32) Schwartz, T.; Hutchison, J. A.; Genet, C.; Ebbesen, T. W. Phys. Rev. Lett. 2011, 106 (19), 196405.

(33) Romero, I.; Aizpurua, J.; Bryant, G. W.; García de Abajo, F. J. Opt. Express 2006, 14 (21), 9988-9999.

(34) Song, P.; Nordlander, P.; Gao, S. J. Chem. Phys. 2011, 134 (7), 074701.

(35) Lindstrom, C. D.; Zhu, X.-Y. Chem. Rev. 2006, 106 (10), 42814300.

(36) Frischkorn, C.; Wolf, M. Chem. Rev. 2006, 106 (10), 42074233.

(37) Hill, R. T.; Mock, J. J.; Hucknall, A.; Wolter, S. D.; Jokerst, N. M.; Smith, D. R.; Chilkoti, A. ACS Nano 2012, 6 (10), 9237-9246.

(38) Kern, J.; Großmann, S.; Tarakina, N. V.; Häckel, T.; Emmerling, M.; Kamp, M.; Huang, J.-S.; Biagioni, P.; Prangsma, J. C.; Hecht, B. Nano Lett. 2012, 12 (11), 5504-5509.

(39) Dulkeith, E.; Morteani, A. C.; Niedereichholz, T.; Klar, T. A.; Feldmann, J.; Levi, S. A.; van Veggel, F. C. J. M.; Reinhoudt, D. N.; Möller, M.; Gittins, D. I. Phys. Rev. Lett. 2002, 89 (20), 203002.

(40) Anger, P.; Bharadwaj, P.; Novotny, L. Phys. Rev. Lett. 2006, 96 (11), 113002.

(41) Zuolaga, J.; Prodan, E.; Nordlander, P. Nano Lett. 2009, 9 (2), 887-891.

(42) Marinica, D. C.; Kazansky, A. K.; Nordlander, P.; Aizpurua, J.; Borisov, A. G. Nano Lett. 2012, 12 (3), 1333-1339.

(43) Esteban, R.; Borisov, A. G.; Nordlander, P.; Aizpurua, J. Nat. Commun. 2012, 3, 825.

(44) Stella, L.; Zhang, P.; García-Vidal, F. J.; Rubio, A.; GarcíaGonzález, P. J. Phys. Chem. C 2013, 117 (17), 8941-8949.

(45) Savage, K. J.; Hawkeye, M. M.; Esteban, R.; Borisov, A. G.; Aizpurua, J.; Baumberg, J. J. Nature 2012, 491 (7425), 574-577.

(46) Scholl, J. A.; García-Etxarri, A.; Koh, A. L.; Dionne, J. A. Nano Lett. 2013, 13 (2), 564-569.

(47) Zuloaga, J.; Prodan, E.; Nordlander, P. ACS Nano 2010, 4 (9), 5269-5276.
(48) Fernández-Domínguez, A. I.; Wiener, A.; García-Vidal, F. J.; Maier, S. A.; Pendry, J. B. Phys. Rev. Lett. 2012, 108 (10), 106802.

(49) Toscano, G.; Raza, S.; Xiao, S.; Wubs, M.; Jauho, A. P.; Bozhevolnyi, S. I.; Mortensen, N. A. Opt. Lett. 2012, 37 (13), 25382540.

(50) Teperik, T. V.; Nordlander, P.; Aizpurua, J.; Borisov, A. G. Phys. Rev. Lett. 2013, 110 (26), 263901.

(51) Marques, M. A. L.; Gross, E. K. U. Annu. Rev. Phys. Chem. 2004, $55,427-455$.

(52) Stratmann, R. E.; Scuseria, G. E.; Frisch, M. J. J. Chem. Phys. 1998, 109 (19), 8218.

(53) de Heer, W. A. Rev. Mod. Phys. 1993, 65 (3), 611-676.

(54) Prodan, E.; Nordlander, P.; Halas, N. J. Nano Lett. 2003, 3 (10), $1411-1415$

(55) Prodan, E.; Nordlander, P.; Halas, N. J. Chem. Phys. Lett. 2003, $368(1-2), 94-101$.

(56) Gunnarson, O.; Lundqvist, B. I. Phys. Rev. B 1976, 13 (10), 4274-4298.

(57) Artuso, R. D.; Bryant, G. W. Phys. Rev. B 2010, 82 (19), 195419.

(58) Salomon, A.; Gordon, R. J.; Prior, Y.; Seideman, T.; Sukharev,

M. Phys. Rev. Lett. 2012, 109 (7), 073002.

(59) Lopata, K.; Neuhauser, D. J. Chem. Phys. 2009, 130 (10), 104707.

(60) Magnier, M.; Aubert-Frécon, M. J. Quant. Spectrosc. Radiat. Transfer 2002, 75 (1), 121-128.

(61) Safronova, M. S.; Arora, B.; Clark, C. W. Phys. Rev. A 2006, 73 (2), 022505.

(62) Winter, H. Phys. Rep. 2002, 367 (5), 387-582.

(63) Nordlander, P.; Tully, J. C. Phys. Rev. B 1990, 42 (9), 55645578 .

(64) Borisov, A. G.; Teillet-Billy, D.; Gauyacq, J. P.; Winter, H.; Dierkes, G. Phys. Rev. B 1996, 54 (23), 17166-17174.

(65) Knoesel, E.; Hertel, T.; Wolf, M.; Ertl, G. Chem. Phys. Lett. 1995, 240 (5-6), 409-416.

(66) Zhu, X.-Y. Annu. Rev. Phys. Chem. 2002, 53, 221-247.

(67) Chulkov, E. V.; Borisov, A. G.; Gauyacq, J. P.; Sánchez-Portal, D.; Silkin, V. M.; Zhukov, V. P.; Echenique, P. M. Chem. Rev. 2006, 106 (10), 4160-4206.

(68) Johnson, P. B.; Christy, R. W. Phys. Rev. B 1972, 6 (12), 43704379.

(69) Kühn, S.; Håkanson, U.; Rogobete, L.; Sandoghdar, V. Phys. Rev. Lett. 2006, 97 (1), 017402.

(70) Carminati, R.; Greffet, J.-J.; Henkel, C.; Vigoureux, J. M. Opt. Commun. 2006, 261 (2), 368-375.

(71) Persson, B. N. J. Chem. Phys. Lett. 1981, 82 (3), 561-565.

(72) Morton, S. M.; Silverstein, D. W.; Jensen, L. Chem. Rev. 2011, 111 (6), 3962-3994.

(73) Galperin, M.; Nitzan, A. Phys. Chem. Chem. Phys. 2012, 14 (26), 9421-9438. 